\title{
Rhamnaceae endémicas del Perú
}

\section{Blanca León ${ }^{1,2}$}

${ }^{1}$ Museo de Historia Natural, Av. Arenales 1256, Aptdo. 14-0434, Lima 14, Perú

2 Plant Resources Center, University of Texas at Austin, Austin TX 78712 EE.UU.

blanca.leon@mail.utexas.edu

\section{Resumen}

La familia Rhamnaceae es reconocida en el Perú por presentar once géneros y 27 especies (Brako \& Zarucchi, 1993; Ulloa Ulloa et al., 2004), entre arbustos, árboles y lianas. Un género, Johnstonia, es endémico del Perú. En este trabajo reconocemos dos especies endémicas en dos géneros. Estas especies endémicas provienen de las regiones Bosques Secos y Desierto Cálido Tropical, desde los 150 hasta los $2200 \mathrm{~m}$ de altitud. Ninguna de las especies endémicas se encuentra representada dentro del Sistema Nacional de Áreas Naturales Protegidas por el Estado.

Palabras claves: Rhamnaceae, Johnstonia, Perú, endemismo, plantas endémicas.

\section{Abstract}

The Rhamnaceae are represented in Peru by eleven genera and 27 species (Brako \& Zarucchi, 1993; Ulloa Ulloa et al., 2004), between shrubs, trees and lianas. One genus, Johnstonia, is endemic to Peru.Here we recognize two endemic taxa in the same number of genera. These endemic species come from Dry Forests and Tropical Costal Desert regions, from 150 to 2200 m elevation. None of these endemic species have been recorded within Peru's protected areas system.

Keywords: Rhamnaceae, Johnstonia, Peru, endemism, endemic plants.

\section{Johnstonia axilliflora (M.C. Johnst.) Tortosa}

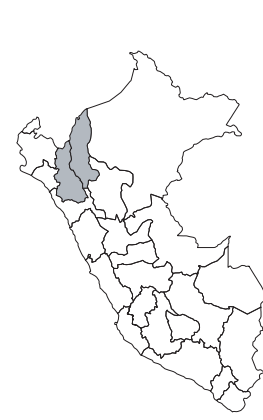

\section{EN, B1a}

Publicación: Novon 15(4): 642. 2005. Colección tipo: D.N. Smith \& R. Vásquez 3396

Herbarios: MO, TEX; AMAZ!, USM!

Nombre común: Desconocido.

Registro departamental: AM, CA. Regiones Ecológicas: BS; $2100 \_2600 \mathrm{~m}$. SINANPE: Sin registro.

Herbarios peruanos: AMAZ (isotipo), USM (isotipo).

Observaciones: Esta liana se conoce de dos localidades, en las cuencas del Crisneja y del Marañón. Habita ambientes xéricos dominados por cactáceas, los cuales no reciben protección oficial alguna. Esta especie fue incluida en Brako \& Zarucchi (1993) en el género Gouania y recientemente transferida al género endémico Johnstonia, de la cual es el tipo (Tortosa, 2005).

\section{Ziziphus piurensis Pilg.}

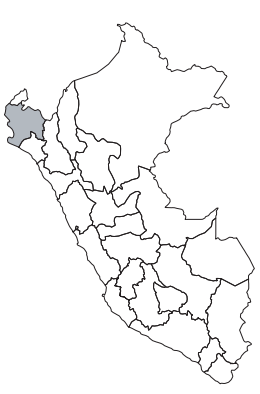

DD

Publicación: Bot. Jahrb. Syst. 54(Beibl. 117): 46. 1916.

Colección tipo: A. Weberbauer 5962

Herbarios: B.

Nombre común: Ebano, palo negro.

Registro departamental: PI.

Regiones Ecológicas: DCT; $150 \mathrm{~m}$.

SINANPE: Sin registro.

Herbarios peruanos: Ninguno.

Observaciones: Árbol de hasta $8 \mathrm{~m}$ de alto conocido de la localidad tipo en el norte del país. Esta especie fue recolectada en 1912 durante un evento «El Niño» en la cuenca del río Piura. Poco se sabe de las características del hábitat de esta especie. Podría ser coespecífica con Ziriphus thyrsiflora de Ecuador y Perú. 